\title{
A INFLUÊNCIA DO ESTADO MENTAL EM ACIDENTES DE TRABALHO: UMA REVISÃO DE LITERATURA
}

\author{
THE INFLUENCE OF MENTAL STATE IN ACCIDENTS AT WORK: \\ A LITERATURE REVIEW
}

\section{Guilherme Valcorte ${ }^{1}$, Jéssica Kelling de Carvalho ${ }^{2}$, Caroline Valcorte de Carvalho ${ }^{3} \mathrm{e}^{4}$}

\section{RESUMO}

$\mathrm{Na}$ atualidade, na qual os trabalhadores têm como exigência a lucratividade, ou seja, o aumento da produção, há um número crescente de casos de exaustão, além de patologias como ansiedade, depressão e o uso de psicotrópicos. Esses fatores, que interferem no estado mental desses trabalhadores, vêm sendo estudados devido à sua associação com a ocorrência de acidentes de trabalho. Esta revisão sistemática tem como objetivo avaliar e discutir a influência do estado mental em acidentes de trabalho. A revisão de literatura foi realizada por meio de buscas de artigos nas bases de dados Pubmed e Scielo e, como critério de inclusão, foram selecionadas publicações nas línguas portuguesa e inglesa. Desta busca, 501 artigos foram obtidos como resultado mediante a utilização das palavras-chave. Após a exclusão conforme critérios, houve a seleção de 6 artigos que posteriormente foram incluídos na presente revisão da literatura, não sendo encontradas novas pesquisas acerca do tema por meio da busca nas referências. Nas publicações selecionadas foram relatadas alterações no estado mental como exaustão, uso de drogas psicotrópicas e sofrimento psicológico grave. Estes estudos mostraram uma relação entre o aumento da incidência de acidentes ocupacionais e o estado mental dos trabalhadores. Essa relação foi analisada considerando bancos de dados de hospitais, pesquisas nacionais e questionários, os quais foram analisados por meio de instrumentos de mensuração. Discutir essa temática é necessário para que os profissionais da área de segurança do trabalho aprendam e saibam lidar com este problema cada vez mais presente no mercado de trabalho.

Palavras-chave: Acidente ocupacional, doença mental, exaustão, medicamento psicotrópico.

\begin{abstract}
Nowadays, which workers demand for profitability, that is, the increase in production, there is an increasing number of exhaustion cases, in addition to pathologies such as anxiety, depression and the use of psychotropics. These factors, which interfere in the workers' mental state, have been studied due to their association with the occurrence of work accidents. This systematic review aims to assess and discuss the influence of mental status in occupational accidents. The literature review was carried out by searching for articles in the Pubmed and Scielo databases, as an inclusion criterion, publications in Portuguese and English were selected. Of this research, 501 articles were obtained as results using the keywords. After exclusion according to criteria, there was a selection of 6 articles, which were subsequently included in the present literature review, and no new research on the topic was found by searching the references. In the selected publications, changes in mental status were reported, such as exhaustion, use of psychotropic drugs and severe psychological distress. These studies showed a relationship between the increased incidence of occupational accidents and

\footnotetext{
${ }^{1}$ Eng. de Segurança do Trabalho e Eng. Florestal, Mestrando do Programa de Pós-graduação em Engenharia Florestal pela Universidade Federal de Santa Maria (UFSM). E-mail: gvalcorte@gmail.com

${ }^{2}$ Graduanda de Medicina da Universidade Federal do Rio Grande (FURG). E-mail: jkellingc@gmail.com

${ }^{3}$ Graduanda de Medicina da Universidade Federal de Santa Maria (UFSM). E-mail: cvalcorte@gmail.com

${ }^{4}$ Doutora em Química pela Universidade Federal de Santa Maria (UFSM). E-mail: kamilakaysercasola@gmail.com
} 
the workers' mental state. This relationship was analyzed considering hospital databases, national surveys and questionnaires, which were analyzed using measurement instruments. Discussing this topic is necessary for professionals in the area of occupational safety to learn and know how to deal with this problem that is increasingly present in the market.

Keywords: Occupational accident, mental illness, exhaustion, psychotropic medication.

\section{INTRODUÇÃO}

Em decorrência da era industrial, tornam-se nítidas as mudanças relacionadas aos costumes e às formas de organização do trabalho e, embora os trabalhadores consigam se beneficiar com os avanços tecnológicos, esta industrialização trouxe como consequência a competitividade e a busca incessante pela lucratividade, o que acaba resultando em pressão para que alcancem metas e aumentem a produtividade. Isso tudo afeta tanto na qualidade de vida quanto na saúde dos trabalhadores (ANTUNES, 2008).

No contexto atual, percebe-se que a exposição dos trabalhadores a riscos físicos, químicos, ergonômicos, psicossociais e/ou biológicos decorrentes da forma que desempenham o seu trabalho pode resultar em doenças e agravos ocupacionais (ARAGÓN et al., 2011), sendo que a doença ocupacional é definida como qualquer doença que de alguma forma foi produzida ou desencadeada pelo exercício do trabalho característico à determinada atividade. Ainda, os agravos associados ao trabalho são relacionados com as doenças e acidentes que afetam a população geral, mas que adquirem características particularmente diferenciadas em certas categorias (BRASIL, 1991).

As altas demandas de trabalho ocasionam uma série de agravos à saúde e acidentes. Por conta disso, a vigilância em saúde do trabalhador tem como objetivo prevenir e identificar estes acidentes precocemente, possibilitando a elaboração de estratégias de intervenção à saúde e melhoria da qualidade de vida do trabalhador (KARINO et al., 2015). Ainda nesta esfera de fatores que são potenciais causadores de acidentes de trabalho, sabe-se que o sofrimento psicológico grave é inespecífico, podendo acarretar em vários sintomas psiquiátricos e constituir uma doença mental grave o suficiente para causar comprometimento moderado a sério no funcionamento social, profissional ou escolar (KESSLER et al., 2002).

A existência de um ambiente laboral equilibrado, que conte com condições para que o trabalhador possa exercer seu oficio com segurança e saúde física e mental mínimas, mostra-se decisiva para a saúde e o bem-estar de homens e mulheres que vivem do trabalho. É sabido que a existência de um ambiente de trabalho saudável influencia o equilíbrio de suas vidas familiares, o desenvolvimento das relações sociais por essas pessoas travadas, o êxito e a produtividade nos estabelecimentos em que laboram e, em última instância, a sociedade em que se inserem (ROCHA; BUSSINGUER, 2016). As doenças ocupacionais quando comparadas aos acidentes de trabalho, causam seis vezes 
mais mortes do que os acidentes de trabalho convencionais (OIT, 2013). Por este motivo, faz-se necessário compreender a doença, examinar o meio ambiente laboral em que se desenvolve e discutir o enfrentamento necessário para mitigá-las (ROCHA; BUSSINGUER, 2016).

Neste seguimento, são cada vez mais comuns problemas relacionados à saúde mental e uso de medicamentos psicotrópicos, pois os mesmos possuem um potencial de causar sonolência e prejudicam o trabalhador nas atividades diárias (PALMER; HARRIS; COGGON, 2008). Como consequência, o excesso de trabalho pode acarretar a fadiga, que é considerada por diversos autores como um esgotamento físico e mental grave e crônico, podendo ser um fenômeno preocupante e de difícil conceituação, interpretação e aferição, pois acaba por nomear um estado global resultante do desequilíbrio interno devido ao sistema de relações do organismo, no qual muitas vezes a alteração de um sistema afeta os demais (OLIVEIRA et al., 2010).

Acerca dos fatores citados, o objetivo deste estudo consiste em realizar uma revisão de literatura a fim de verificar a influência do estado mental em acidentes de trabalho e analisar se doenças psicológicas e drogas psicotrópicas estão diretamente relacionadas aos mesmos.

\section{MÉTODOS}

Com o objetivo de produzir essa revisão sistemática, foram pesquisados artigos que apontassem dados sobre a influência do estado mental em acidentes de trabalho. Assim, realizaram-se buscas nas bases de dados Pubmed e Scielo com a finalidade de comprovar a influência do estado mental fragilizado no aumento da prevalência de acidentes ocupacionais. Para tanto, foram usadas as palavras-chave: (“work accidents" OR "workplace accidents" OR "accidents at work" OR "workplace Injuries” OR “occupational accidents”) AND (“self-esteem” OR “mental illness” OR “mental disorders" OR “mental health conditions").

Para seleção dos artigos encontrados foi utilizado como método de exclusão: 1) Duplicidade dos estudos nas diferentes bases de dados; 2) Leitura dos títulos e identificação de estudos não condizentes com o tema; 3) Revisões sistemáticas referentes ao tema; 4) Estudos descritivos referentes ao tema; 5) Artigos publicados anteriormente ao ano 2000. Assim, foram selecionados artigos que realizaram estudos de caso controle, coorte ou transversal referentes ao tema, apresentando amostras e grupos controles.

\section{RESULTADOS}

Como exposto na figura 1, posteriormente às buscas nas bases de dados foram encontrados 501 artigos. Destes, descartou-se 20 que apresentaram duplicidade entre as bases e, por meio da leitura de títulos, foram rejeitados 450 que não se adequaram ao tema, restando 31 artigos elegíveis. 
A partir disso, foi lido o resumo dos estudos elegíveis e excluídos 5 referentes à revisões sistemáticas, 10 estudos descritivos e 10 que foram publicados em data anterior ao ano 2000. Assim, restaram 6 artigos que serão utilizados para a elaboração dessa revisão.

Figura 1 - Fluxograma de identificação e seleção dos artigos para revisão sistemática

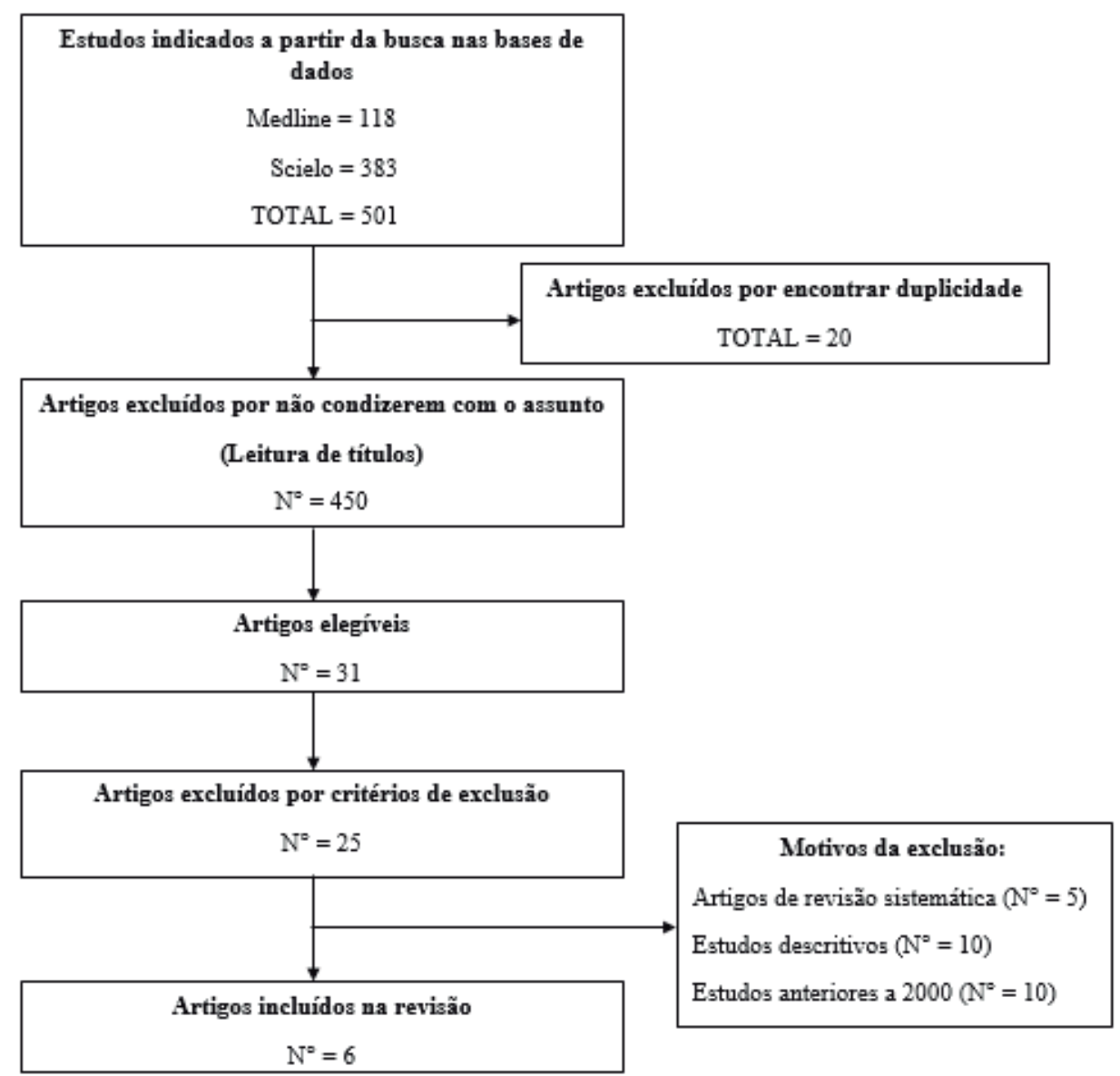

Fonte: Construção dos Autores.

Quanto às características gerais dos artigos selecionados, as datas de publicação variaram de 2008 a 2019, com desenhos transversais $(83,33 \%)$ ou de coorte (16,66\%), originados de 5 diferentes países: Brasil (33,33\%), Grã-Bretanha (16,66\%), Japão (16,66\%), EUA (16,66\%) e Austrália (16,66\%), como demonstrado na tabela 1.

Tabela 1 - Características dos estudos sobre a influência do estado mental em acidentes de trabalho.

\begin{tabular}{lcc}
\hline \multicolumn{1}{c}{ Autor (Ano) } & Desenho & Local \\
\hline Celikkalp e Dilek (2019) & Transversal & Brasil \\
\hline Hilton e Whiteford (2010) & Transversal & Brasil \\
Kim (2008) & Transversal & EUA \\
\hline Palmer et al., (2014) & Caso-controle aninhado em coorte & Grã-Bretanha \\
Soares et al., (2018) & Transversal & Austrália \\
Tei-Tominaga e Nakanishi (2018) & Transversal & Japão \\
\hline
\end{tabular}

Fonte: Construção dos Autores. 
As amostras variaram de 101.855 a 108 pessoas, com população total de 202.201 trabalhadores. As pesquisas utilizaram banco de dados e, além disso, as informações obtidas foram analisadas por meio de instrumentos de mensuração como a escala de sonolência de Epworth, a escala de carga de trabalho, a escala de Likert e a escala K6.

Conforme as pesquisas referenciadas (CELIKKALP e DILEK, 2019; HILTON e WHITEFORD, 2010; KIM, 2008; PALMER et al., 2014; SOARES et al. 2018; TEI-TOMINAG e NAKANISHI, 2018), as seis indicaram um aumento da prevalência de acidentes de trabalho vinculados ao estado mental fragilizado dos trabalhadores.

Dentre os principais achados dos artigos, são citados estados mentais que influenciam na incidência de acidentes de trabalho, como sonolência decorrente de horas extras, turnos extensos ou a prática de mais de um trabalho (CELIKKALP e DILEK, 2019; SOARES et al. 2018; TEI-TOMINAG e NAKANISHI, 2018); doenças psiquiátricas, como o sofrimento psicológico grave, depressão ou ansiedade (HILTON e WHITEFORD, 2010; KIM, 2008; PALMER et al., 2014) e utilização de medicamentos psicotrópicos (PALMER et al., 2014), como exposto na tabela 2.

Tabela 2 - Características dos estudos sobre a influência do estado mental em acidentes de trabalho.

\begin{tabular}{|c|c|c|c|c|}
\hline Autor (Ano) & Objetivo (s) do estudo & $\begin{array}{c}\text { Coleta de Dados/ } \\
\text { Instrumento de mensuração }\end{array}$ & Amostra & Principais achados \\
\hline $\begin{array}{l}\text { Celikkalp e } \\
\text { Dilek (2019) }\end{array}$ & $\begin{array}{l}\text { Determinar os motivos } \\
\text { de acidentes de traba- } \\
\text { lho em uma amostra de } \\
\text { enfermeiros turcos. }\end{array}$ & $\begin{array}{l}\text { Questionário, utilizando para o } \\
\text { estudo e categorização do estado } \\
\text { mental dos enfermeiros a escala de } \\
\text { sonolência de Epworth, assim como } \\
\text { a escala de carga de trabalho. }\end{array}$ & 108 enfermeiros & $\begin{array}{l}68,5 \%(\mathrm{n}=74) \text { dos enfermeiros } \\
\text { sofreram um acidente ocupacional, } \\
\text { sendo que a maioria trabalhava ho- } \\
\text { ras extras. Dessa forma, a carga de } \\
\text { trabalho e o sono foram associados } \\
\text { com a ocorrência destes acidentes. }\end{array}$ \\
\hline $\begin{array}{l}\text { Hilton e } \\
\text { Whiteford } \\
(2010)\end{array}$ & $\begin{array}{l}\text { Este estudo investiga } \\
\text { associações entre } \\
\text { sofrimento psíquico e } \\
\text { acidentes de trabalho }\end{array}$ & $\begin{array}{l}\text { Questionário de Saúde e Desem- } \\
\text { penho no Trabalho (HPQ), sendo } \\
\text { o estresse psicológico quantificado } \\
\text { pela escala de Kessler } 6 \text { (K6). }\end{array}$ & $\begin{array}{l}60.556 \text { funcionários } \\
\text { em período integral }\end{array}$ & $\begin{array}{l}\text { Os funcionários com sofrimento } \\
\text { psicológico moderado e alto pos- } \\
\text { suem uma propensão aumentada à } \\
\text { ocorrência de acidentes de trabalho } \\
\text { (OR = } 2,3 \text { e } 2,6 \text {, respectivamente). } \\
\text { Como a prevalência de sofrimento } \\
\text { psíquico moderado é aproximada- } \\
\text { mente o dobro do sofrimento psí- } \\
\text { quico alto, o sofrimento moderado } \\
\text { tem consequentemente um maior } \\
\text { impacto no local de trabalho. }\end{array}$ \\
\hline Kim (2008) & $\begin{array}{l}\text { Examinar se o sofri- } \\
\text { mento psicológico gra- } \\
\text { ve (SPD) está associado } \\
\text { a lesões ocupacionais } \\
\text { entre os funcionários } \\
\text { dos EUA. }\end{array}$ & $\begin{array}{l}\text { Dados do National Health Interview } \\
\text { Survey (NHIS) 2000-2003, o estado } \\
\text { psicológico foi medido através da } \\
\text { escala de socorro psicológico de } \\
\text { Kessler (K6) }\end{array}$ & $\begin{array}{l}101.855 \text { pessoas em- } \\
\text { pregadas nos EUA. }\end{array}$ & $\begin{array}{l}\text { A prevalência de lesões ocupacio- } \\
\text { nais foi } 37 \% \text { maior em trabalha- } \\
\text { dores com SPD, sugerindo que o } \\
\text { SPD é responsável por uma maior } \\
\text { probabilidade de lesões ocupacio- } \\
\text { nais entre funcionários dos EUA. }\end{array}$ \\
\hline
\end{tabular}


Palmer et al.

\section{Estabelecer uma}

relação entre doenças

mentais e drogas psico-

trópicas com lesões no

local de trabalho.

$\begin{array}{ll}\text { Soares et al. } & \text { Identificar a influência } \\ \text { (2018) } & \text { do estresse físico e psi- } \\ & \text { cossocial e dos fatores } \\ & \text { relacionados à saúde na } \\ & \text { ocorrência de acidentes } \\ & \text { de trabalho. }\end{array}$

Banco de dados do Clinical Practice

Research Datalink (CPRD), que rela-

ciona os pacientes que consultaram

por lesões no local de trabalho e que

também consultaram por doenças

mentais ou que obtiveram receita de

medicamentos psicotrópicos. A base

de dados foi consultada de 1987 a

2009.

9612 pessoas, 1602 consultaram decorrente de lesão no local de trabalho e 8010 controles.

Dados da Pesquisa Nacional de Saúde (NHS) realizada no Brasil em 2013 em 2013, avaliados com base no German Health Update 2010.

$23 \%$ dos indivíduos que apresentaram lesões ocupacionais já haviam consultado decorrente de doenças psiquiátricas e $21 \%$ haviam obtido a prescrição de um medicamento psicotrópico. Conforme a pesquisa, problemas de saúde mental aumentam a chance de lesões em $44 \%$ e a prescrição de psicotrópicos aumentam o risco de lesões em 57\%.

Tei-Tominaga A influência do am-

e Nakanishi biente de trabalho na (2018) ocorrência de acidentes ocupacionais.

\author{
Questionários. O ambiente favorável \\ no local de trabalho foi avaliado \\ através da escala de Likert, já a \\ escala K6 foi utilizada para avaliar o \\ psicológico dos enfermeiros.
} $2,8 \%(n=987)$ esteve envolvido em um acidente de trabalho. Verificou-se que o trabalho em período integral, durante longas horas e com três ou mais empregos são variáveis que apontam para uma propensão mais significativa a acidentes de trabalho.

$\begin{array}{ll}35.248 \text { trabalha- } & 2,8 \% \text { (n=987) esteve envolvido em } \\ \text { dores } & \text { um acidente de trabalho. Verifi- } \\ & \text { cou-se que o trabalho em período } \\ & \text { integral, durante longas horas e } \\ & \text { com três ou mais empregos são } \\ & \text { variáveis que apontam para uma } \\ & \text { propensão mais significativa a } \\ & \text { acidentes de trabalho. } \\ \text { de } 11 \text { hospitais. } & 5 \% \text { (n=42) dos participantes } \\ & \text { sofreram acidentes no local de } \\ & \text { trabalho, sendo que 34\% desses } \\ & \text { (n=15) trabalhavam horas extras } \\ & \text { e 28\% (n=11) trabalhavam em } \\ & \text { dias de folga mais de uma vez } \\ & \text { por mês, mostrando assim que há } \\ & \text { associação significativa de lesões } \\ & \text { no trabalho com a média de horas } \\ & \text { extras trabalhadas. }\end{array}$

Fonte: Construção dos Autores.

\section{DISCUSSÃO}

De acordo com Palmer et al., (2014), pacientes com problemas de saúde mental são comumente tratados com remédios psicotrópicos e essas drogas são geralmente prescritas para doentes mentais. Portanto, o estudo comparou os riscos de acidentes de trabalho em indivíduos sem problemas de saúde mental (PSM), pacientes com problemas de saúde mental mas sem receita médica e aqueles com problema de saúde mental e com receita médica. Com base nos resultados, pode-se observar que a utilização de drogas psicotrópicas contribui para algum tipo de evento de lesões no local de trabalho. Tal conclusão reafirma a questão principal do presente artigo, mostrando que o estado mental prejudicado e a utilização de drogas psicotrópicas relacionadas a seu tratamento influenciam nos acidentes de trabalho de maneira negativa, ou seja, tanto a doença mental quanto os fármacos utilizados em seu tratamento aumentam a ocorrência de acidentes de trabalho.

Ainda, após análise Celikkalp e Dilek (2019) observaram as condições do trabalho e puderam verificar que os enfermeiros da Turquia sofriam acidentes de trabalho devido à grande demanda de 
trabalho, como horas extras, trabalho sobrecarregado, vários turnos e trabalhar à noite. Com isso, os autores puderam observar que o cenário afetava negativamente a qualidade de sono dos trabalhadores o que, consequentemente, culminava em acidentes de trabalho com maiores escores médios na escala de sonolência de epworth (ESSE). Este resultado reitera a importância de um ambiente de trabalho adequado, visando condições salubres e que possibilitem ao trabalhador exercer sua atividade laboral de maneira segura, tendo em vista a segurança não apenas física como, de maneira igualmente importante, sua segurança mental. A sobrecarga de trabalho culmina na diminuição da concentração e expertise devido ao cansaço mental propiciado pela má qualidade de sono. Dessa forma, os trabalhadores se tornam suscetíveis à ocorrência de erros que culminam em acidentes laborais, sendo esses evitáveis diante de uma carga horária adequada de trabalho, primando pelo descanso físico e mental.

Em concordância com os autores anteriores, Tei-Tominaga e Nakanishi (2018) escreveram acerca da influência de ambientes de trabalho éticos e de apoio em termos de aspectos sociais relacionados ao trabalho na experiência de acidentes ou lesões laborais em enfermeiros hospitalares no Japão. Com os resultados de uma análise de regressão multivariada, os autores observaram que as pessoas que trabalhavam mais de 4 horas extras por semana e aqueles que trabalhavam nos dias de folga mais de uma vez no mês e com um ambiente exclusivo no local de trabalho, apresentavam experiências de acidentes de trabalho ou lesões. Além disso, o ambiente exclusivo resultou num elevado risco de acidentes nos enfermeiros, pelo fato de estes estarem mais acostumados com o local.

Ademais, conforme relatado por Kim (2008) com base em formulários, trabalhadores diagnosticados com sofrimento psíquico eram os mais propensos a sofrer fraturas, queimaduras e contusões, entre outras lesões. Já Soares et al. (2018) conseguiram verificar que há a chance de o estresse estar relacionado ao número de empregos que o trabalhador possua, já que muitos possuem três ou mais empregos e tal fator, quando comparado àqueles com apenas um emprego, acaba validando a tese de que pessoas que trabalham em vários locais têm mais chances de desencadear estresse no trabalho. Isso demonstra que, tanto empregadores quanto empregados devem se atentar de maneira a fornecer e promover ambientes de trabalho adequados não só na esfera física como também na esfera social destes locais de trabalho, não ultrapassando seus limites fisiológicos em sua jornada de trabalho e buscando compor um ambiente harmônico para os trabalhadores.

Por fim, Hilton e Whiteford (2010) relatam que o sofrimento psicológico aumenta o risco de falhas no trabalho e diminui a ocorrência de sucesso nestes locais. Além disso, neste estudo foi possível comprovar que trabalhadores expostos a sofrimento psicológico moderado e estresse psicológico alto estão relacionados significativamente a um aumento de acidentes de trabalho. Diante dos fatos supracitados, fica clara a relação da segurança laboral com a saúde mental do trabalhador, demonstrando a relação direta do sofrimento psicológico com o aumento da incidência de acidentes. 


\section{CONCLUSÃO}

A partir desta revisão de literatura, conseguimos concluir que a ocorrência de acidentes de trabalhado aos trabalhadores pode ser desencadeada por algumas características, como o estado mental fragilizado em que o trabalhador se encontra, bem com o ambiente que esta pessoa tem de realizar suas tarefas diárias, além de horas extras de trabalho, sonolência, doenças como depressão ou ansiedade e até mesmo a utilização de medicamentos psicotrópicos.

A importância de estudos com esta temática é cada vez mais relevante, pois conseguimos ver que os números de acidentes ocupacionais relacionados à saúde mental do trabalhador estão crescendo significativamente.

\section{REFERÊNCIAS}

ANTUNES, R. Adeus ao trabalho: ensaio sobre as metamorfoses e a centralidade do mundo do trabalho. 12. ed. Rio de Janeiro: Cortez Editora, 2008.

ARAGÓN, A; PARTANEN T; FELKNOR S; CORRIOLS M. Social determinants of workers' health in Central America. International Journal of Occupational and Environmental Health. Philadelphia, UK, v. 17, n. 3, p. 230-237, Jun. 2011. DOI: 10.1179/107735211799041986.

BRASIL. Ministério da Previdência Social. Lei no 8.213, de 24 de julho de 1991. Dispõe sobre os Planos de Benefícios da Previdência Social e dá outras providências. DOU, Brasília, 14 Ago 1991.

CELIKKALP, U; DILEK, F. Factors affecting the occupational accident rates among nurses. Revista da Escola de Enfermagem da USP. São Paulo, v. 53, e03524, 2019 . Epub Dec 02, 2019. DOI: 10.1590/ s1980-220x2018049703524.

HILTON, M. F; WHITEFORD, H. A. Associations between psychological distress, workplace accidents, workplace failures and workplace successes. International Journal of Occupational and Environmental Health. Berlin, DEU, v. 83, n. 8, p. 923-933, dez. 2010. DOI: 10.1007/s00420010-0555-x.

KARINO, M. E; FELLI, V. E. A; SARQUIS, L. M. M; SATANA, L. L; SILVA, S. R; TEIXEIRA, R. C. Cargas de trabalho e descargas dos trabalhadores de enfermagem de um hospital-escola. Ciência, Cidade e Saúde. Maringá, v. 14, n. 2, p. 1011-1018, nov. 2015. DOI: 10.4025/cienccuidsaude.v14i2.21603. 
KESSLER R. C; ANDREWS G; COLPE L. J; HIRIPI E; MROCZEK D. K; NORMAND S. L. Short Screening Scales to Monitor Population Prevalences and Trends in Non-Specific Psychological Distress. Psychological Medicine. Rio de Janeiro, v. 68, n. 1. 2002. DOI: 10.1017/s0033291702006074

KIM J. Psychological distress and occupational injury: findings from the National Health Interview Survey 2000-2003. Journal Preventive Medicine e Public Health. 2008. v. 41. n. 3, p. 200-207. DOI: 10.3961/jpmph.2008.41.3.200.

OLIVEIRA, J. R. S; VIGANO, M. G; LUNARDELLI, C. F; CANÊO, L. C; JUNIOR, E. G. Fadiga no trabalho: como o psicólogo pode atuar?. Psicologia em estudo. Maringá, v. 15, n. 3, p. 1413-7372, set. 2010.

ORGANIZAÇÃO INTERNACIONAL DO TRABALHO. A prevenção das doenças profissionais. Genebra: OIT, 2013. Disponível em: https://bit.ly/35DyzFG. Acesso em: Janeiro de 2020.

PALMER, K. T; D'ANGELO, S; HARIS, E. C; LINAKER, C; COGGON, D. The role of mental health problems and common psychotropic drug treatments in accidental injury at work: a case-control study. Occupational and Environmental Medicine. v. 71. n. 5, mar. 2014. DOI: 10.1136/ oemed-2013-101948.

PALMER, K. T, HARRIS, E. C, COGGON, D. Chronic health problems and risk of accidental injury in the workplace: a systematic literature review. Occupational and Environmental Medicine. v. 65. n. 11, p. 757-764, nov. 2008. DOI: 10.1136/oem.2007.037440.

ROCHA, S. H; BUSSINGUER, E. C. A. A invisibilidade das doenças mentais ocupacionais no mundo contemporâneo do trabalho. Pensar. Fortaleza, v. 21, n. 3, p. 1104-1122, set. 2016.

SOARES, S. M; GELMINI, S; BRANDÃO, S. S. S; SILVA, J, M. C. Workplace accidents in brazil: analysis of physical and psychosocial stress and health-related factors. Revista de Administração Mackenzie, São Paulo, v. 19, n. 3, jul. 2018. DOI: 10.1590/1678-6971/eramg170131.

TEI-TOMINAGA, M; NAKANISHI, M. The Influence of Supportive and Ethical Work Environments on Work-Related Accidents, Injuries, and Serious Psychological Distress among Hospital Nurses. International Journal of Occupational and Environmental Health. v. 15. n. 2, p. 240, jan. 2018. DOI: $10.3390 /$ ijerph15020240. 
